\title{
Feasibility of Flat Panel Detector CT in Perfusion Assessment of Brain Arteriovenous Malformations: Initial Clinical Experience
}

\author{
(D) M. Garcia, (D)T.W. Okell, (DM. Gloor, (D) M.A. Chappell, DP. Jezzard, (D) O. Bieri, and (D).V. Byrne
}

\begin{abstract}
SUMMARY: The different results from flat panel detector CT in various pathologies have provoked some discussion. Our aim was to assess the role of flat panel detector CT in brain arteriovenous malformations, which has not yet been assessed. Five patients with brain arteriovenous malformations were studied with flat panel detector CT, DSC-MR imaging, and vessel-encoded pseudocontinuous arterial spin-labeling. In glomerular brain arteriovenous malformations, perfusion was highest next to the brain arteriovenous malformation with decreasing values with increasing distance from the lesion. An inverse tendency was observed in the proliferative brain arteriovenous malformation. Flat panel detector $\mathrm{CT}$, originally thought to measure blood volume, correlated more closely with arterial spin-labeling-CBF and DSC-CBF than with DSC-CBV. We conclude that flat panel detector CT perfusion depends on the time point chosen for data collection, which is triggered too early in these patients (ie, when contrast agent appears in the superior sagittal sinus after rapid shunting through the brain arteriovenous malformation). This finding, in combination with high data variability, makes flat panel detector CT inappropriate for perfusion assessment in brain arteriovenous malformations.
\end{abstract}

ABBREVIATIONS: $A S L=$ arterial spin-labeling; $\mathrm{bAVM}=$ brain arteriovenous malformation; $\mathrm{FPD}=$ flat panel detector; $\mathrm{pn}=$ perinidal; $\mathrm{rCBF}=\mathrm{relative} \mathrm{CBF} ; \mathrm{rCBV}=$ relative CBV

G omerular brain arteriovenous malformations (bAVMs) are characterized by pathologic vessels without intermingled normal brain tissue, whereas proliferative bAVMs are identified by normal brain tissue between dispersed abnormal vessels. The pattern of involved vessels composes the angioarchitecture of the lesion, while the hemodynamic character has been less well-studied.

The redirection of blood flow to the nidus of the AVM at the expense of perilesional tissues is known as "vascular steal" 1 and

Received August 28, 2016; accepted after revision December 1.

From the Division of Diagnostic and Interventional Neuroradiology (M. Garcia), and Division of Radiological Physics (M. Gloor, O.B.), Department of Radiology Clinic for Radiology and Nuclear Medicine, University of Basel Hospital, Basel, Switzerland; and Nuffield Department of Surgical Sciences and Department of Neuroradiology (M. Garcia, J.V.B.) and Centre for Functional Magnetic Resonance Imaging of the Brain (FMRIB Centre) (T.W.O., M.A.C., P.J.), Nuffield Department of Neurosciences, and Institute of Biomedical Engineering (M.A.C.), Department of Engineering, University of Oxford, Oxford, United Kingdom.

Thomas W. Okell and Michael A. Chappell are authors of a pending US patent licensed to Siemens (Erlangen, Germany) relating to the maximum a posteriori (MAP) processing technique used in this study. The royalties went to the coauthors (T.W.O. and M.A.C.).

Please address correspondence to Meritxell Garcia, MD, MSc, Division of Diagnostic and Interventional Neuroradiology, Department of Radiology, Clinic for Radiology and Nuclear Medicine, University Hospital Basel, Petersgraben 4, 4031 Basel, Switzerland; e-mail: meritxell.garcia@usb.ch

http://dx.doi.org/10.3174/ajnr.A5091 has been studied by xenon-enhanced CT, ${ }^{2}{ }^{133} \mathrm{Xe}$ inhalation with conventional angiography, ${ }^{3} \mathrm{CTP},{ }^{4} \mathrm{PET},{ }^{5,6}$ SPECT, ${ }^{7}$ DSC-MR imaging, ${ }^{8}$ and arterial spin-labeling (ASL). ${ }^{9,10}$ However, these techniques have been inconsistent in demonstrating perinidal hypoperfusion.

Flat panel detector CT (FPD-CT) is a novel technique for brain perfusion assessment, providing parenchymal blood volume maps. ${ }^{11-18}$ The aim of this study was to investigate the value of FPD-CT in bAVMs, which has not been assessed, yet, to our knowledge. FPD-CT data were compared with DSC-MR imaging and ASL-MR imaging. ${ }^{19,20}$

\section{Case Series}

Five patients with large $(>3 \mathrm{~cm}$ ) bAVMs ( 4 glomerular bAVMs, patients $1-4 ; 1$ proliferative bAVM, patient 5) underwent FPD-CT and MR imaging with DSC and ASL. None of the patients had undergone prior treatment except patient 1, whose large $\mathrm{BAVM}$ had received partial treatment with 1 coil and $0.5 \mathrm{~mL}$ of a 1:1 n-BCA/lipiodol mixture with the goal of reducing arteriovenous shunting in a semiemergent situation. The study protocol was approved by the local ethics committee (Oxford University). Written consent was obtained from all patients.

AJNR Am J Neuroradiol 38:735-39 Apr 2017 www.ajnr.org 


\section{MATERIALS AND METHODS}

\section{Flat Panel Detector CT}

FPD-CT was performed on a biplane FPD-angiography system (Axiom Artis dBA; Siemens, Erlangen, Germany). Eighty milliliters of iopamidol (370 mg iodine/mL, Niopam 370; Bracco, Milan, Italy) was applied intravenously (injection rate, $5 \mathrm{~mL} / \mathrm{s}$; injection pressure, $300 \mathrm{psi}$ ). The acquisition protocol consisted of 2 rotations: a mask run and a contrast-enhanced run. We manually started the latter ${ }^{14}$ as soon as contrast opacification of the superior sagittal sinus was observed, assuming that this time point represented a steady-state of contrast in the brain parenchyma for ideal collection of the fill run data. The parameters for each rotation run were the following: acquisition time, 8 seconds; matrix, $616 \times$ 480 ; projection on a $30 \times 40 \mathrm{~cm}$ flat panel; total angle, $200^{\circ}$; $0.5^{\circ}$ /frame with a total of 400 frames. After the C-arm return of the run mask to its starting position, 2D-DSA images at a rate of 2 images per second were acquired for documentation of the contrast passage through the cerebral vasculature. ${ }^{17,18}$ The acquisition time was approximately 40 seconds. ${ }^{14}$

The reconstructed mask run and fill run were subtracted from each other. The steady-state value was calculated from an automated histogram analysis of the vessel tree. ${ }^{20}$ Further postprocessing provided an isotropic volume dataset.

\section{MR Imaging Perfusion}

DSC and ASL perfusion were performed on a 3T Tim Verio system (Siemens). An axial T1WI contrast-enhanced sequence served as a reference (acquisition time, 1 minute 46 seconds; TR/TE, 215/2.48 ms; 15 sections; section thickness, $4 \mathrm{~mm}$; matrix, $208 \times 320$ ).

DSC perfusion imaging was performed with a gradient-echo echo-planar imaging sequence during the first pass of a $20-\mathrm{mL}$ intravenous bolus of gadoteridol or gadobenate dimeglumine (ProHance or MultiHance; Bracco) at an injection rate of $5 \mathrm{~mL} / \mathrm{s}$ followed by $40 \mathrm{~mL}$ of saline. Imaging parameters were as follows: TR/TE, 1500/30 ms; section thickness, $4 \mathrm{~mm}$; voxel size, $1.6 \times$ $1.2 \times 4 \mathrm{~mm}$; matrix, $152 \times 202$; acquisition time, 1 minute 23 seconds. Fifty measurements were obtained, with the injection occurring at the third image. Postprocessing (syngo.MR Neuro Perfusion Engine; Siemens) generated relative $\mathrm{CBF}(\mathrm{rCBF})$ and relative $\mathrm{CBV}$ (rCBV) maps. The arterial input function was selected in the middle cerebral artery in the hemisphere opposite to the bAVM.

Quantitative ASL perfusion was performed with a recently described vessel-encoded pseudocontinuous ASL sequence, ${ }^{19,20}$ allowing encoding of the selected arteries (right/left internal carotid artery, right/left vertebral artery) within the labeling plane on 3DTOF-MRA and hence providing artery-specific absolute CBF maps. For subsequent vessel-encoded pseudocontinuous ASL acquisitions, 8 paired encoding cycles were obtained as previously described. ${ }^{19-22}$

The total CBF map was calculated by summing the CBF maps from each artery. A single arrival-time (ASL-arrival time) map, representing the time required for the blood to travel from the neck to the brain tissue, was calculated by summing the arrival-time maps from each artery, weighted by the relative CBF contributed by that artery.

\section{Data Postprocessing and Analysis}

After converting the FPD-CT, DSC-MR imaging, ASL-MR imaging, and T1WI contrast-enhanced DICOM data into NIfTI files
(FSL, www.fmrib.ox.ac.uk/fsl), we extracted the brain parenchyma by using the FSL Brain Extraction Tool (http://fsl.fmrib. ox.ac.uk/fsl/fslwiki/BET). Each dataset was coregistered to the T1weighted contrast-enhanced image. Six WM and 2 GM ROIs, which served as a reference, and corresponding contralateral mirror ROIs were drawn as masks on the T1-weighted contrast-enhanced image:

Masks 1 and $2(\mathrm{WM})$ : perinidal $(\mathrm{pn}=0.2-1 \mathrm{~cm}$ away from the nidus).

Masks 3 and 4 (WM): vicinity ( $1-3 \mathrm{~cm}$ away from the nidus). Masks 5 and 6 (WM): remote ( $>3 \mathrm{~cm}$ away from the nidus).

Mask 7 (GM): putamen.

Mask 8 (GM): thalamus.

To minimize partial volume effects, we excluded tissue in the adjacent $2 \mathrm{~mm}$ from the bAVM (Fig 1).

The respective set of masks was overlaid onto each single perfusion map. The mean and SD of the masks were evaluated. The interhemispheric perfusion values (ratios) for each corresponding pair of masks were used for data analysis. Assessment of perfusion correlations among all modalities was performed by aggregating each corresponding mask of all patients and analyzed with the Pearson correlation coefficient $(r)$. The correlation interpretation scheme was the following: none or weak $(0.0-0.3)$, moderate $(0.3-0.7)$, strong $(0.7-0.9)$, and very strong $(0.9-1)$.

\section{RESULTS}

The resolution of the FPD-CT proved to be sufficient and artifactfree on visual assessment, and the masks drawn on T1-weighted contrast-enhanced imaging showed a good correspondence with the raw FPD-CT data (Fig 2). The perfusion distribution for all patients is shown in Fig 3.

\section{Patients 1 to 4}

The interhemispheric perfusion ratios for FPD-CT, ASL, and DSC were highest in the pn regions, with decreasing ratios with increasing distance from the bAVM. The putamen and the thalamus (reference), which were both remote from the glomerular bAVMs, showed similar values in both hemispheres, with interhemispheric ratios close to 1 (Fig 3).

\section{Patient 5}

The perfusion ratios of the proliferative bAVM (FPD-CT, ASL$\mathrm{CBF}$, and DSC-rCBF) showed a slightly increasing tendency from the perinidal to the remote brain tissue, with markedly less abrupt perfusion differences between the adjacent ROIs (Fig 3), whereas almost no changes between the different WM masks could be observed for DSC-rCBV.

In patient 5 , the thalamus, which was included within the bAVM, showed a ratio below 1 for FPD-CT, DSC-rCBF, and ASL$\mathrm{CBF}$. The ratio of the putamen (not included in the bAVM) was markedly positive for FPD-CT, slightly positive for DSC-rCBF and around 1 for ASL-CBF and DSC-rCBV (data not shown).

\section{Data Variability}

When we aggregated all masks of patients 1-4 (excluding the only outlier, pn 1 of patient 3), variability of the perfusion data was by far higher for FPD-CT than for ASL. FPD-CT data variability was similar to that of DSC-rCBF and slightly higher than that obtained 


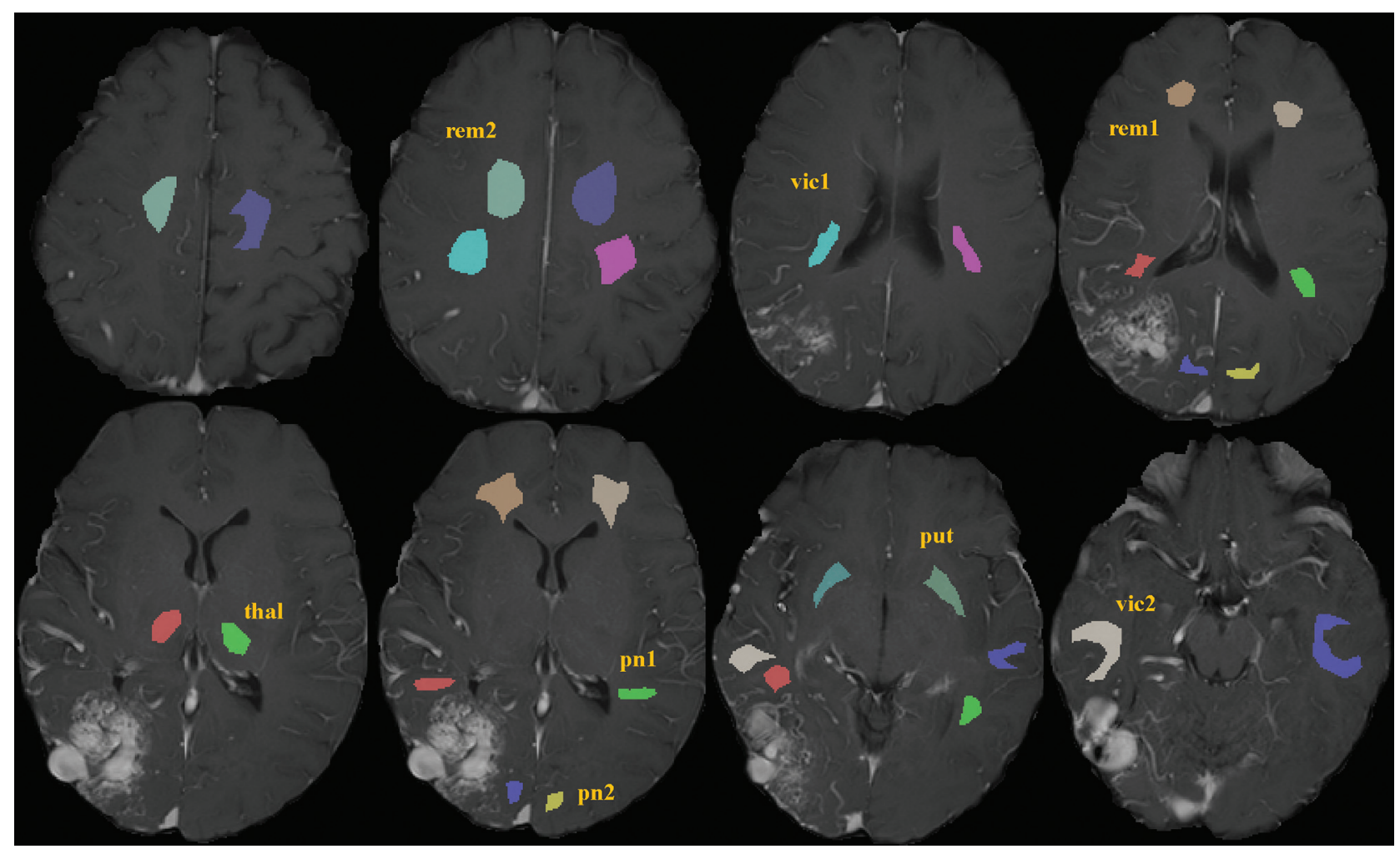

FIG 1. Examples of the different WM (pnl/pn2, vicl/vic2, reml/rem2) and GM masks (put, thal) selected on the TIWI contrast-enhanced sequence. Each mask has been labeled in either of the 2 hemispheres. The set of masks was loaded on each perfusion map separately, which had previously been coregistered to the TIWI contrast-enhanced dataset. Except for some of the GM masks, almost all masks were drawn as a volume, which explains their delineation on several consecutive sections. Note the different color for the respective mask in the contralateral hemisphere, because the software did not allow the use of the same color for the respective opposite mask. pn indicates perinidal; vic, vicinity; rem, remote; thal, thalamus; put, putamen.

T1wCE

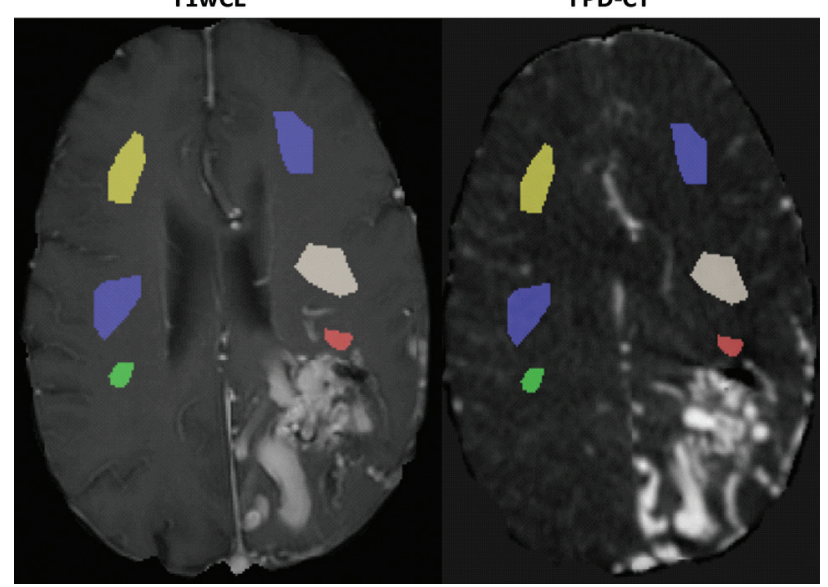

FIG 2. When superimposing the red mask representing the ipsilateral pnl ROI previously drawn in the TIWI contrast-enhanced dataset (TIwCE; left) on the FPD-CT perfusion dataset (right), a good overlap without contamination from bAVM tissue could be observed.

from DSC-rCBV (Table). When we compared the perfusion data of the pn masks only, perfusion data variability was lower for FPD-CT than for DSC.

\section{Correlations between and within Modalities}

The highest correlation was observed between FPD-CT and ASLCBF $(r=0.60)$, followed by a moderate correlation between
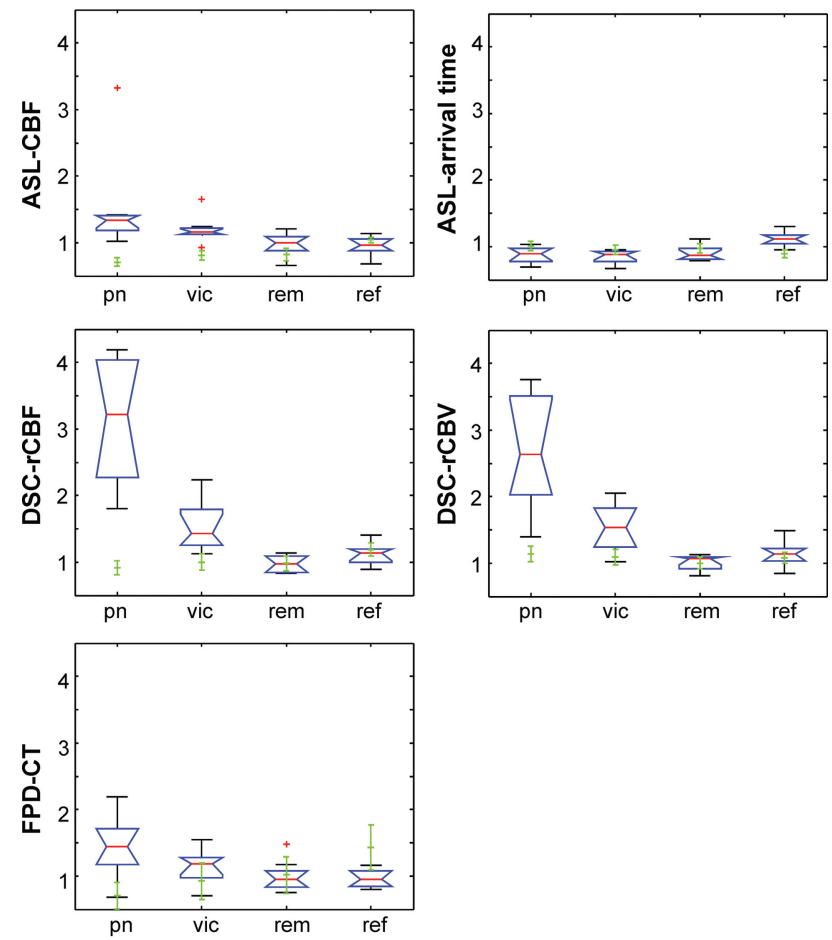

FIG 3. Box-and-whisker plots for patients 1-4. The boxes show the median, lower, and upper quartiles. Outliers are displayed as red crosses. The mean and SD across the pixels of each ROI of patient 5 are represented in green. ref indicates reference. 
FPD-CT and DSC-rCBF ( $r=0.47)$. The correlation between FPD-CT and DSC-rCBV was weak $(r=0.36)$, and it was negative between FPD-CT and ASL-arrival time $(r=-0.42)$ (Fig 4).

\section{DISCUSSION}

Imaging modalities assessing perfusion around bAVMs have provided different findings, which may be ascribed to the limitations of the techniques. The good correlation between parenchymal blood data from FPD-CT and CBV obtained from CTP has been demonstrated in ischemia. ${ }^{12-15}$ However, recently, it has been shown that in vasospasm, FPD-CT perfusion is both CBV- and CBF-weighted. ${ }^{17,18}$ Furthermore, parenchymal blood volume maps obtained from CTA source images ${ }^{23}$ have been reported to be either CBV- or CBF-weighted, depending on the order of CTA/ CTP data acquisition. ${ }^{24}$

In this study, the glomerular bAVMs showed a perinidal hyperperfusion, with a tendency to normalization with increasing distance from the nidus. The perinidal hyperperfusion would, at first glance, disagree with the "vascular steal" theory. However, it

$\begin{aligned} & \text { Coefficients of variation aggregating all masks, excluding the } \\
& \text { outlier (pn } \mathbf{1} \text { of patient 3), across all patients }\end{aligned}$
\begin{tabular}{lc}
\multicolumn{1}{c}{ Modality } & Coefficient of Variation \\
\hline FPD-CT & $21.2 \%$ \\
ASL-CBF & $11.0 \%$ \\
ASL-arrival time & $12.3 \%$ \\
DSC-rCBF & $21.3 \%$ \\
DSC-rCBV & $17.8 \%$ \\
\hline
\end{tabular}

is difficult to believe that a vascular steal is absent in the presence of a high-flow lesion. Probably, in these glomerular bAVMs, a certain amount of vascular steal was present but not apparent due to the ability of the brain to maintain $\mathrm{CBF}$ within a certain range of perfusion pressure.

In contrast, the regionally more extensive hypoperfusion in the proliferative bAVM suggests that the brain parenchyma may have already reached a chronic state of hypoperfusion, resembling cerebral proliferative angiopathy. ${ }^{25}$

ASL appeared to be superior to DSC-MR imaging and FPD$\mathrm{CT}$, showing the lowest data variability, in addition to its lack of need for contrast. A higher and similar variability was observed between FPD-CT and DSC-MR imaging, except in the pn ROI, where data variability was lower for FPD-CT, presumably due to magnetic susceptibility artifacts in the perinidal DSC data.

In the proliferative bAVM, in contrast to ASL-CBF, DSCrCBV, and DSC-rCBF, the interhemispheric ratio for FPD-CT of the putamen, which was at some distance from the bAVM, showed a marked positive interhemispheric ratio. These divergent observations might be attributed to partial volume effects with FPD-CT, which, in the more remote tissue, might exceed the MR imaging artifacts, the latter being more pronounced next to the bAVMs. However, more studies with larger patient pools are required to explain this finding.

In contrast to FPD-CT studies performed on ischemia ${ }^{12-15}$ and on vasospasm ${ }^{17,18}$ in our study on bAVMs, perfusion correlated best with CBF. The strongest correlation was found with ASL-CBF. This observation is because the start of data collection
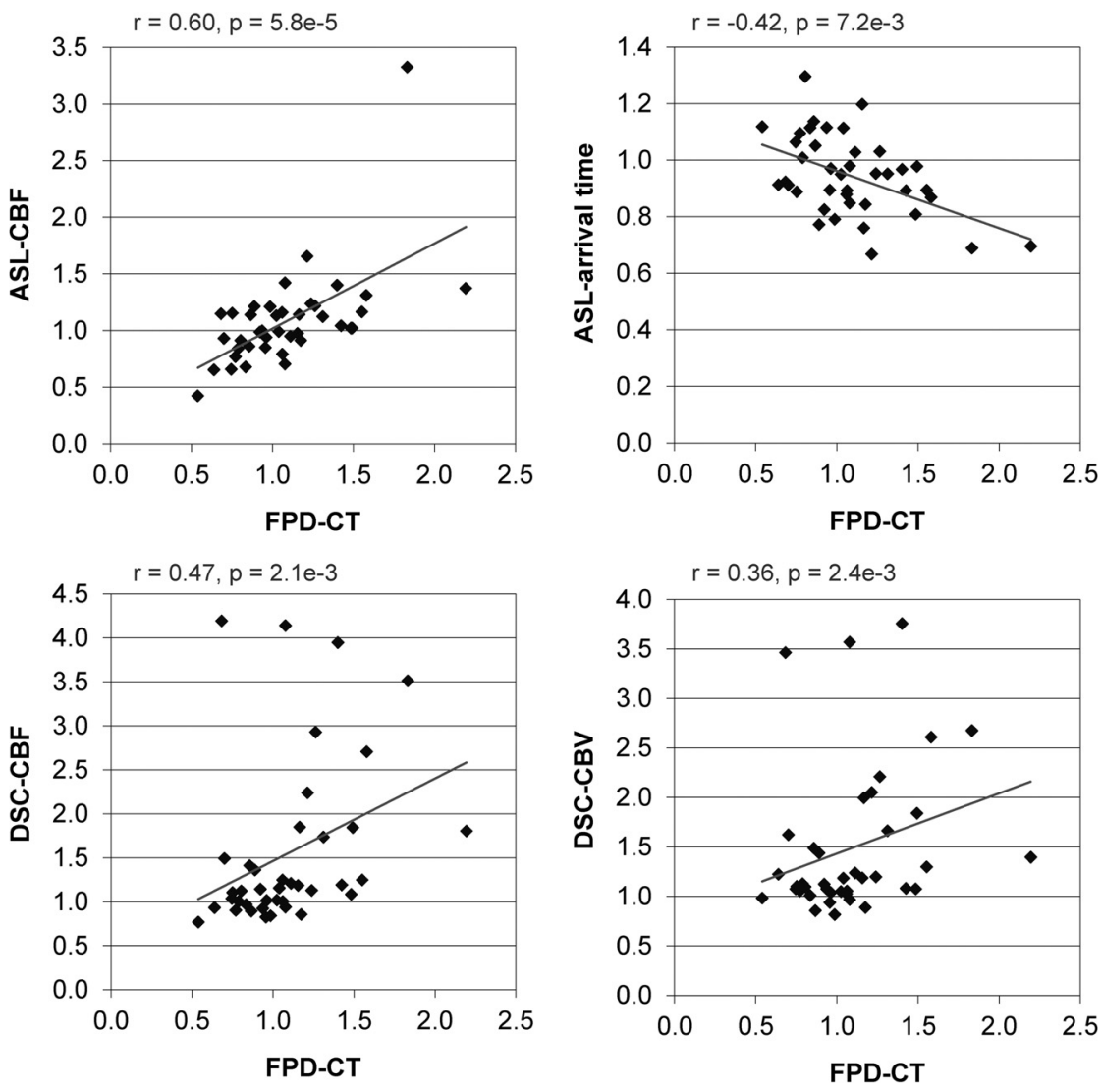

FIG 4. Correlations between different parameters for all patients. The squares represent the ratio for a single pair of masks. is based on the visualization of the superior sagittal sinus, which may not reflect a "true" steady-state because the rapid transit of contrast through bAVMs may cause early filling of the superior sagittal sinus. This may result in data collection ahead of time, thus explaining the strong correlation of FPD-CT with CBF and the negative correlation with ASL-arrival time, because the FPD-CT signal will not have reached its maximum in brain regions where blood arrives later.

To date, no perfusion method can be regarded as a reference in bAVMs. Although our main aim was not to compare different perfusion techniques, all of the 3 methods investigated showed similar perfusion trends. Nevertheless, the obtained results cannot be extrapolated to other patient groups due to the unique hemodynamic properties of bAVMs. Therefore, it is not surprising that bAVMs show perfusion patterns different from those of other vascular pathologies of the brain.

A comparison of FPD-CT and CTP would also have been of interest. However, because in our institution patients with bAVMs are not routinely investigated with CTP, the additional radiation 
would not have been justified. Furthermore, for the additional assessment of ASL-MR imaging, DSC-MR imaging seemed a more appropriate reference though we are aware that DSC-MR imaging is not the optimal perfusion reference, either.

Perfusion data obtained with FPD-CT appears to comprise a variable contribution from flow and volume, depending on the type of vascular lesion and the trigger time point chosen for data collection. ${ }^{26}$ In bAVMs, FPD-CT correlated best with CBF-assessed MR imaging, and the different perfusion patterns between bAVM subtypes might be attributed to differences in the angioarchitecture. To accurately calculate either CBF or CBV with FPD-CT is very challenging in high-flow lesions. In addition to the heterogeneous characteristics of bAVMs and the high data variability, FPD-CT is not appropriate for an acceptable perfusion assessment in bAVMs.

Disclosures: Tom W. Okell—UNRELATED: Grant: The Royal Academy of Engineering and the Dunhill Medical Trust, Comments: These funding bodies provided grants for my research position, which enabled me to undertake the work described in this article*; Royalties: Siemens, Comments: royalties received for an exclusive license to a pending US patent relating to the maximum a posteriori Bayesian vessel-encoding analysis technique used in this study. Michael A. Chappell—UNRELATED: Patents (Planned, Pending, or Issued): Siemens, Comments: Principal Investigator relating to vessel-encoded ASL MRI data analysis; Royalties: FSL, Comments: commercial licensing of the FMRIB Software Library for fMRI data analysis. Peter Jezzard_-UNRELATED: Patents (Planned, Pending, or (ssued): Siemens, Comments: Patent application on data analysis, licensed to Siemens. Oliver Bieri-UNRELATED: Grants/Grants Pending: Siemens, ${ }^{*}$ Comments: I receive research support from Siemens Healthineers (formerly Siemens). James $V$. Byrne-UNRELATED: Provision of Writing Assistance, Medicines, Equipment, or Administrative Support: Siemens, Comments: loan to department of analysis software and computer by Siemens. *Money paid to the institution.

\section{REFERENCES}

1. Taylor CL, Selman WR, Ratcheson RA. Steal affecting the central nervous system. Neurosurgery 2002;50:679-88; discussion 688-89 CrossRef Medline

2. Van Roost D, Schramm J. What factors are related to impairment of cerebrovascular reserve before and after arteriovenous malformation resection? A cerebral blood flow study using xenon-enhanced computed tomography. Neurosurgery 2001;48:709-16; discussion 716-17 Medline

3. Young WL, Pile-Spellman J, Prohovnik I, et al. Evidence for adaptive autoregulatory displacement in hypotensive cortical territories adjacent to arteriovenous malformations: Columbia University AVM Study Project. Neurosurgery 1994;34:601-10; discussion 610-11 CrossRef Medline

4. Kim DJ, Krings T. Whole-brain perfusion CT patterns of brain arteriovenous malformations: a pilot study in 18 patients. AJNR Am J Neuroradiol 2011;32:2061-66 CrossRef Medline

5. Fink GR. Effects of cerebral angiomas on perifocal and remote tissue: a multivariate positron emission tomography study. Stroke 1992;23:1099-105 CrossRef Medline

6. Tyler JL, Leblanc R, Meyer E, et al. Hemodynamic and metabolic effects of cerebral arteriovenous malformations studied by positron emission tomography. Stroke 1989;20:890-98 CrossRef Medline

7. Ueda M, Kamiya T, Sakamoto S, et al. Abnormally distributed regional cerebral blood flow in brain malformations detected by single photon emission computed tomography [in Japanese]. Rinsho Shinkeigaku 1997;37:99-105 Medline

8. Guo WY, Wu YT, Wu HM, et al. Toward normal perfusion after radiosurgery: perfusion MR imaging with independent component analysis of brain arteriovenous malformations. AJNR Am J Neuroradiol 2004;25:1636-44 Medline
9. Fiehler J, Illies T, Piening M, et al. Territorial and microvascular perfusion impairment in brain arteriovenous malformations. AJNR Am J Neuroradiol 2009;30:356-61 CrossRef Medline

10. Wolf RL, Wang J, Detre JA, et al. Arteriovenous shunt visualization in arteriovenous malformations with arterial spin-labeling MR imaging. AJNR Am J Neuroradiol 2008;29:681-87 CrossRef Medline

11. Kamran M, Nagaraja S, Byrne JV. C-arm flat detector computed tomography: the technique and its application in interventional neuro-radiology. Neuroradiology 2010;52:319-27 CrossRef Medline

12. Mordasini P, El-Koussy M, Brekenfeld C, et al. Applicability of tableside flat panel detector CT parenchymal cerebral blood volume measurement in neurovascular interventions: preliminary clinical experience. AJNR Am J Neuroradiol 2012;33:154-58 CrossRef Medline

13. Struffert T, Deuerling-Zheng Y, Kloska S, et al. Cerebral blood volume imaging by flat detector computed tomography in comparison to conventional multislice perfusion CT. Eur Radiol 2011;21:882-89 CrossRef Medline

14. Struffert T, Deuerling-Zheng Y, Engelhorn T, et al. Feasibility of cerebral blood volume mapping by flat panel detector CT in the angiography suite: first experience in patients with acute middle cerebral artery occlusions. AJNR Am J Neuroradiol 2012;33:618-25 CrossRef Medline

15. Blanc R, Pistocchi S, Babic D, et al. Intravenous flat-detector CT angiography in acute ischemic stroke management. Neuroradiology 2012;54:383-91 CrossRef Medline

16. Fiorella D, Turk A, Chaudry I, et al. A prospective, multicenter pilot study investigating the utility of flat detector derived parenchymal blood volume maps to estimate cerebral blood volume in stroke patients. J Neurointerv Surg 2014;6:451-56 CrossRef Medline

17. Kamran M, Downer J, Corkill R, et al. Non-invasive assessment of vasospasm following aneurysmal SAH using C-arm FDCT parenchymal blood volume measurement in the neuro-interventional suite: technical feasibility. Interv Neuroradiol 2015;21:479-89 CrossRef Medline

18. Kamran M, Byrne JV. C-arm flat detector computed tomography parenchymal blood volume imaging: the nature of parenchymal blood volume parameter and the feasibility of parenchymal blood volume imaging in aneurysmal subarachnoid haemorrhage patients. Neuradiology 2015;57:937-49 CrossRef Medline

19. Okell TW, Chappell MA, Kelly ME, et al. Cerebral blood flow quantification using vessel-encoded arterial spin labeling. J Cereb Blood Flow Metab 2013;33:1716-24 CrossRef Medline

20. Wong EC. Vessel-encoded arterial spin-labeling using pseudocontinuous tagging. Magn Reson Med 2007;58:1086-91 CrossRef Medline

21. Chappell MA, Okell TW, Jezzard P, et al. Vascular territory image analysis using vessel encoded arterial spin labeling. Med Image Comput Comput Assist Interv 2009;12(pt 2):514-21 Medline

22. Chappell MA, Okell TW, Payne SJ, et al. A fast analysis method for non-invasive imaging of blood flow in individual cerebral arteries using vessel-encoded arterial spin labelling angiography. Med Image Anal 2012;16:831-39 CrossRef Medline

23. Sharma M, Fox AJ, Symons $S$, et al. CT angiographic source images: flow- or volume-weighted? AJNR Am J Neuroradiol 2011;32:359-64 CrossRef Medline

24. Dorn F, Liebig T, Muenzel D, et al. Order of CT stroke protocol (CTA before or after CTP): impact on image quality. Neuroradiology 2012; 54:105-12 CrossRef Medline

25. Fierstra J, Spieth S, Tran L, et al. Severely impaired cerebrovascular reserve in patients with cerebral proliferative angiopathy. J Neurosurg Pediatr 2011;8:310-15 CrossRef Medline

26. Kloska SP. CT angiography source images with modern multisection CT scanners: attention to technical principles is crucial. Cerebrovasc Dis 2011;32:94 CrossRef Medline 